\title{
Novel neutron reflectometer SOFIA at J-PARC/MLF for in-situ soft-interface characterization
}

\author{
Koji Mitamura ${ }^{1}$, Norifui L Yamada ${ }^{2}$, Hidenori Sagehashi ${ }^{2}$, Naoya Torikai ${ }^{3}$, Hiroshi Arita ${ }^{4}$, Masami Terada $^{1}$, \\ Motoyasu Kobayashi ${ }^{1}$, Setsuo Sato ${ }^{2}$, Hideki Seto ${ }^{2}$, Shinji Goko ${ }^{5}$, Michihiro Furusaka ${ }^{5}$, Tatsuro Oda ${ }^{6}$, \\ Masahiro Hino ${ }^{7}$, Hiroshi Jinnai ${ }^{1,8,9}$ and Atsushi Takahara ${ }^{1,4,8,9}$
}

\begin{abstract}
Structural characterization of interfaces composed of soft materials (soft interfaces) helps in understanding their physical behavior. Neutron reflectometry is one of the most powerful tools to characterize interfacial structures with spatial resolution in nanometers. We have installed a novel horizontal-type time-of-flight neutron reflectometer SOFIA (SOFt Interface Analyzer) at the Japan Proton Accelerator Research Complex. The instrument is capable of accepting two downward neutron beams, at $2.2^{\circ}$ and $5.7^{\circ}$ to horizontal, which dedicate neutron reflectivity (NR) measurements over a wide range of neutron momentum transfer $q\left(q=(4 \pi / \lambda) \sin \theta\right.$, where $\lambda$ and $\theta$ are wavelength and incident angle, respectively). The accuracy of NR up to $q=6 \mathrm{~nm}^{-1}$ was confirmed by measuring deuterated polystyrene (d-PS) thin films on a silicon (Si) wafer and multilayers of cadmium stearate prepared by the Langmuir-Blodgett method. NR at the deuterium oxide $\left(D_{2} \mathrm{O}\right) / \mathrm{Si}$ disk showed specular reflection down to $10^{-6}-10^{-7}$ and $q$ up to $2.0 \mathrm{~nm}^{-1}$ along the perpendicular to the sample surface, which improved the precise analysis of swollen polyelectrolyte brush structure at the $\mathrm{D}_{2} \mathrm{O}$ interface. Then, time-resolved in-situ NR measurements were carried out at 1-min intervals to observe interfacial mixing of d-PS on the PS brush surface during $398 \mathrm{~K}$ annealing, demonstrating that nonequilibrium behavior at the interfaces can be analyzed on the order of minutes.
\end{abstract}

Polymer Journal (2013) 45, 100-108; doi:10.1038/pj.2012.156; published online 5 September 2012

Keywords: in-situ characterization; solid/liquid interfaces; neutron reflectometer; polymer brushes; soft interfaces

\section{INTRODUCTION}

It is well known that materials at an interface or on a surface sometimes show behaviors different from their bulk states because of interfacial asymmetry. In particular, interfaces composed of soft materials such as polymers and surfactants, often called soft interfaces, exhibit complex structural and physical features, because their macromolecules often show hierarchical structures; they can form assemblies such as crystals, spherulites and micelles in thin films, and structurally fluctuate over wide time and spatial scales. To understand the relationship between structural and physical features in soft interfaces, it is important to characterize interfacial structures over wide spatial $\left(10^{-1}-10^{4} \mathrm{~nm}\right)$ and time ranges (picoseconds-days or years).

$\mathrm{X}$-rays and neutrons are often employed as probes to evaluate static and dynamical aspects of soft interfaces. For example, X-ray or neutron reflectometry is used to obtain static structural information of the interfaces with nanometer resolution. ${ }^{1}$ X-ray photon correlation spectroscopy allows us to obtain dynamical information over millisecond ranges with nanometer-scale resolution. ${ }^{2,3}$ The neutron spin echo technique is a powerful tool to investigate very fast dynamics from picosecond to nanosecond ranges. ${ }^{4}$

Among the above methods, neutron reflectometry has one particular advantage: the large difference between the scattering length density (SLD) of hydrogen and deuterium makes it possible to dramatically enhance the contrast between hydrogenated and deuterated species in soft interfaces via deuterium labeling. ${ }^{5}$ For reflectivity measurements with a monochromatic beam, an incident angle $(\theta)$ scan is required to observe reflectivity over a wide range of neutron momentum transfer $q=(4 \pi / \lambda) \sin \theta$, where $\lambda$ is the wavelength. On the other hand, a wide-wavelength band is available with the time-offlight (TOF) method using pulsed neutrons, because the wavelength of neutrons depends on their velocity. This allows us to perform in situ, time-resolved measurements of soft materials over a wide $q$ range. Such time-resolved neutron reflectivity (NR) measurements have been attempted at overseas facilities to investigate the swelling behavior of polymers under solvent vapor, interfacial mutual mixing between hydrogenated (h-) and deuterated (d-) molecules, and dewetting

${ }^{1}$ Japan Science and Technology, ERATO Takahara Soft Interfaces Project, Fukuoka, Japan; ${ }^{2}$ Institute of Material Structure Science, High Energy Accelerator Research Organization (KEK), Ibaraki, Japan; ${ }^{3}$ Department of Chemistry for Materials, Graduate School of Engineering, Mie University, Mie, Japan; ${ }^{4}$ Department of Chemistry and Biochemistry, Graduated School of Engineering, Kyushu University, Fukuoka, Japan; ${ }^{5}$ Department of Mechanical and Intelligent System Engineering, Graduate School of Engineering, Hokkaido University, Hokkaido, Japan; ${ }^{6}$ Department of Nuclear Engineering, Graduate School of Engineering, Kyoto University, Kyoto, Japan; 7 Kyoto University Research Reactor Institute, Kumatori, Osaka, Japan; ${ }^{8}$ Institute for Materials Chemistry and Engineering, Kyushu University, Fukuoka, Japan and ${ }^{9}$ nnternational Institute for Carbon-Neutral Energy Research (WPI-I2CNER), Kyushu University, Fukuoka, Japan

Correspondence: Professor A Takahara, Institute for Materials Chemistry and Engineering, Kyushu University, 744 Motooka, Nishi-ku, Fukuoka 819-0395, Japan. E-mail: takahara@cstf.kyushu-u.ac.jp

Received 23 June 2012; revised 23 July 2012; accepted 24 July 2012; published online 5 September 2012 
behavior of polymers on solid surfaces. ${ }^{6-8}$ In these experiments, it took 5-10 min to accumulate statistically sufficient reflected neutrons to generate NR plots, which implies that rapid structural changes within 5-10 min, if any have occurred, may not have been resolved. A shorter accumulation time is preferred to follow the diffusion and intermixing behavior of polymer chains at surfaces or interfaces.

Recently, a new-generation neutron facility with an mega-watt (MW)-class proton accelerator has been constructed in the Materials and Life Science Facility (MLF) at the Japan Proton Accelerator Research Complex (J-PARC, Tokai, Japan). A $25 \mathrm{~Hz}$ pulsed neutron beam is available at MLF and, so far, the proton beam current is $74 \mu \mathrm{A}$ $(220 \mathrm{~kW})$, although it will be upgraded to $333 \mu \mathrm{A}(1 \mathrm{MW})$ in the near future. The neutron flux of each pulse is currently the highest worldwide, even though J-PARC is not yet at full-power operation. There are 23 beamlines at MLF, and beamline 16 (BL16) is dedicated to NR measurements. Thus, the powerful pulsed neutron beam of MLF is very attractive for in-situ measurements. In this beamline, a neutron reflectometer with horizontal geometry, ARISA-II, was installed in December 2008. The main components of the ARISA reflectometer were relocated from the Neutron Science Laboratory (KENS), High Energy Accelerator Research Organization (KEK), Tsukuba, Japan. ${ }^{9}$

Knowledge of the mobile structure at the free-standing surface, as well as at the interface, is also essential to understanding the properties of soft materials such as surfactants, colloids and biological lipid membrane layers on a liquid surface. As free liquid surfaces or interfaces are horizontal, the neutron beam has to be guided vertically downward onto the samples. J-PARC uses white neutrons as a source, so NR with a certain $q$ range can be obtained without changing the scattering angle, which enables us to cover a wider $q$ range than that obtainable using monochromatic incident neutrons. For example, the incident neutron beam at $2.2^{\circ}$ relative to the horizontal and a wavelength of $0.20-0.88 \mathrm{~nm}$ gives specular reflectivity in a $q$ range of $0.54-2.41 \mathrm{~nm}^{-1}$. Nevertheless, our aim is to obtain NR over much wider $q$ ranges than existing analogous equipment, because NR at $q$ ranges higher than $3 \mathrm{~nm}^{-1}$ is necessary for the precise analysis of a small structure at free liquid-vapor and liquid-liquid interfaces. One possible way to increase the $q$ range of the instrument is to vary the incident neutron beam to the specimen. At the BL16 beamline, two downward neutron beams with tilt angles of $2.2^{\circ}$ and $5.7^{\circ}$ are available. Unfortunately, ARISA-II cannot use one of these two beams $\left(5.7^{\circ}\right)$ because of its insufficient specimen travel range. ${ }^{9}$ Thus, a new reflectometer, SOFIA (SOFt Interface Analyzer), was recently designed to utilize both $2.2^{\circ}$ and $5.7^{\circ}$ beams, and has been installed at the BL16 beamline, replacing the existing ARISA-II, from which it has evolved..$^{10,11}$ NR up to $q=6.24 \mathrm{~nm}^{-1}$ can be obtained using the $5.7^{\circ}$ beam, although the maximum measurable $q$ depends on the minimum reflectivity that can be measured and the background from the instrument and sample. This paper describes the design principle, performance and validity of the SOFIA reflectometer using representative examples of soft interfaces.

\section{DESIGN PRINCIPLE OF SOFIA AT BL16}

Figure 1 shows an outline of the BL16 beamline and SOFIA. At BL16, two neutron beamlines with tilt angles of $2.2^{\circ}$ and $5.7^{\circ}$ are transported through supermirror guides over distance $L$ from the moderator in the range $2.3<L<7.0 \mathrm{~m}$. After the guide tubes, the neutrons pass through a disk chopper at $L=7.5 \mathrm{~m}$ and a T0 chopper at $L=8.4 \mathrm{~m}$, which rotate in synchronization with neutron pulses and open for a certain time after the neutron pulse (details of energy shaping will be described below). As a result of a neutron's wavelength depending on its velocity, that is, the time after the pulse, the choppers remove neutrons with unnecessary wavelengths for background reduction. The disk chopper stops relatively slow neutrons in both beamlines with gadolinium paint, and the T0 chopper stops very fast neutrons with a 300 -mm-thick inconel block. The choppers are utilized for both beamlines: the upper parts are for the $2.2^{\circ}$ beamline, the lower parts are for the $5.7^{\circ}$ beamline. In addition, a $\mathrm{NiC}$ mirror on a thin $\mathrm{Si}$ substrate is placed at $L=14.7 \mathrm{~m}$. This mirror totally reflects very slow neutrons that cannot be stopped by the choppers.

Figure 2a illustrates energy shaping using the choppers and $\mathrm{NiC}$ mirror. The proton beam generates pulsed white neutrons every $40 \mathrm{~ms}$. One pulse period corresponds to a single frame. After energy shaping, neutrons with wavelength $\lambda$ in the range $0.11<\lambda<0.88 \mathrm{~nm}$ are available at a repetition rate of $25 \mathrm{~Hz}$. When the rotation rate of the disk chopper switches to $12.5 \mathrm{~Hz}$, the aperture's open period becomes twice that in the single-frame mode and slower neutrons up to $1.76 \mathrm{~nm}$ can pass through the choppers (Figure 2b); this is the double-frame mode operation. On the other hand, the double-frame mode wastes adjacent neutron pulses to extend neutron intervals to $80 \mathrm{~ms}$. In the double-frame mode, therefore, the accumulation time required to obtain reasonable statistics would be longer than that in the single-frame mode. The advantage of the double frame mode, however, is that a wider range of momentum transfer $q=(4 \pi / \lambda) \sin \theta$ can be obtained at a fixed incident angle $\theta$, which would be useful for time-slicing measurements within minute intervals.

In single-frame mode, $\mathrm{NR}$ at low and high $q$ ranges is independently measured with different incident angles $\theta$ by tilting the sample stage and then they are combined to generate an NR curve, because the broad $q$ range cannot be covered by one fixed incident angle. For example, when $\theta$ is $1.0^{\circ}$ using $\lambda=0.11-0.88 \mathrm{~nm}$ neutrons, the observable $q$ range for NR is limited to $0.25-2.0 \mathrm{~nm}^{-1}$, which does not include the critical value of momentum transfer $q_{\mathrm{c}}$ for total reflection. Therefore, additional NR measurement at lower $\theta$ is also carried out by changing the tilt angle of the sample stage to complete the reflectivity curve in a wide $q$ range. On the other hand, the double-frame mode can afford reflectivity measurements with a broad $q$ range using one incident angle due to a wide wavelength band of $0.11-1.76 \mathrm{~nm}$. A practical measurement example is described later.

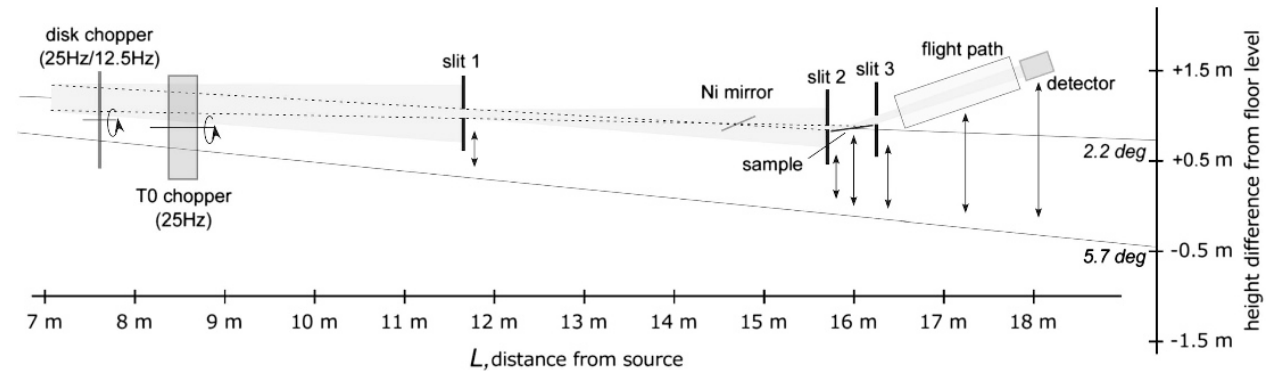

Figure 1 Schematic side view of SOFIA reflectrometer installed in BL16 at MLF in J-PARC. 
a

Single frame mode

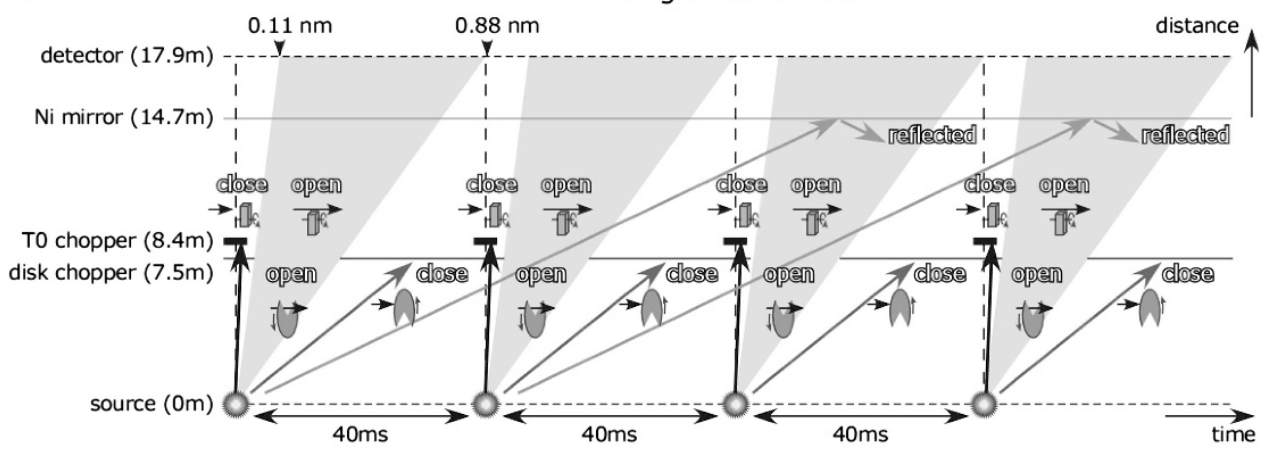

b

Double frame mode

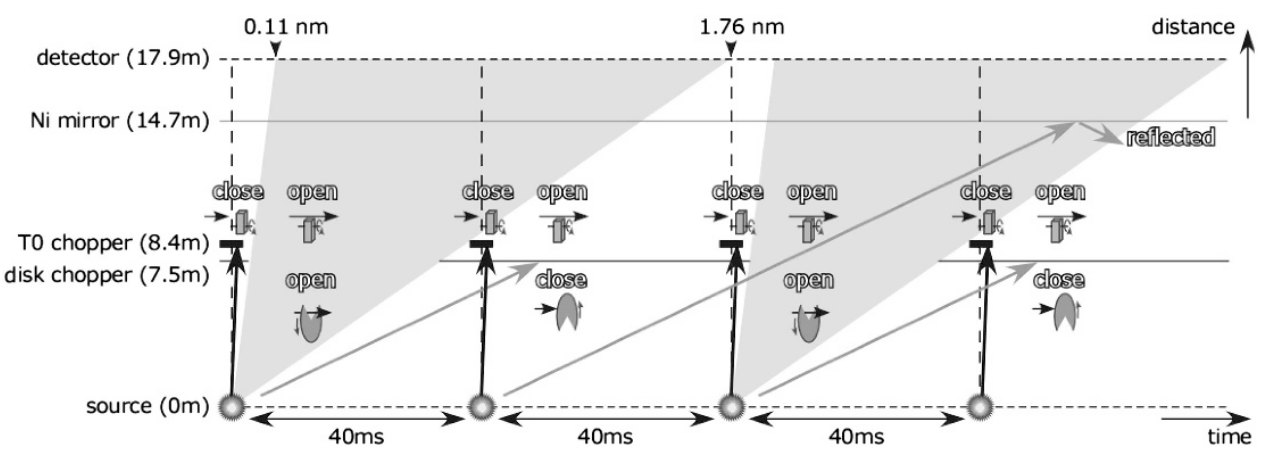

Figure 2 Relationship between rotating rate of disk chopper and available time-of-flight neutrons by (a) single-frame mode and (b) double-frame mode.

After passing the two choppers, the neutrons are collimated with two 4-blade slits made of sintered $\mathrm{B}_{4} \mathrm{C}$ at $L=11.6$ and $15.7 \mathrm{~m}$. The collimated neutrons then irradiate a sample at $L=15.9 \mathrm{~m}$. The neutrons reflected by the sample pass through the third slit at $L=16.1 \mathrm{~m}$ and are finally detected on a two-dimensional scintillation counter at $L=17.9 \mathrm{~m}$, which consists of an A R3239 photomultiplier tube (Hamamatsu Photonics K. K., Hamamatsu, Japan) with a ZnS/ ${ }^{6} \mathrm{LiF}$ scintillator (Ohyo Koken, Fussa, Japan). All the equipment is mounted on stages ( $z$-stage) with translational swivel stages and goniometer to adjust vertical positions and tilt angles of the slits, sample and detector. The travel ranges of $z$-stages are long enough to utilize both the $5.7^{\circ}$ and $2.2^{\circ}$ beamlines. Use of a high-incident-angle beam is one of the key advantages of the SOFIA reflectometer over ARISA-II. The two beamlines and the supermirrors together enable us to measure reflectivity up to $q=6 \mathrm{~nm}^{-1}$ for free liquid surfaces. In addition, the sample stage accelerates and decelerates quite smoothly, and is equipped with an active vibrational isolator (AVI-150LP, JRS Scientific Instruments and Table Stable Ltd., Zwillikon, Switzerland).

Figure 3a displays a contour plot of direct beam intensity at the $2.2^{\circ}$ beamline in the single-frame mode at a $220 \mathrm{~kW}$ proton beam power. The vertical and horizontal axes represent the vertical position on the two-dimensional detector and the TOF $t$, respectively. The beam is collimated vertically to irradiate a $60 \mathrm{~mm} \times 40 \mathrm{~mm}$ footprint at an incident angle of $0.3^{\circ}$ with a beam divergence of $2 \%$; the beam size at the sample position and detector is $0.21 \mathrm{~mm} \times 40 \mathrm{~mm}$ and $0.48 \mathrm{~mm} \times 70 \mathrm{~mm}$, respectively. The full width at half maximum of the intensity profile projected vertically is $0.73 \mathrm{~mm}$, which is consistent with the spatial resolution of the detector (about $1 \mathrm{~mm}$ ).

Figure $3 \mathrm{~b}$ shows the intensity profile projected on the TOF with a time bin of $0.1 \mathrm{~ms}$, in which TOF $t$ is converted to wavelength $\lambda$ using the following distance relation between the neutron source and the detector $L^{\prime}(17.9 \mathrm{~m})$ :

$$
\lambda=3.956 \times 10^{-7}\left(\mathrm{~m}^{2} / \mathrm{s}\right) \times t / L^{\prime} .
$$

This profile shows a Maxwellian peak around $0.25 \mathrm{~nm}$ and an intensity decrease with increasing $\lambda$. However, reflectivity generally increases with decreases in $q$ (or increases in $\lambda$ ). As these two effects compensate for each other, the statistics for obtaining the reflection profile at a fixed incident angle are generally better for the long wavelength neutrons than that at the Maxwellian peak. This is why double-frame mode is adaptable for reflectivity measurements even though neutron flux at long wavelengths is very low. On the other hand, the statistics of the reflection data drastically decreases with $\lambda$ for $\lambda<0.25 \mathrm{~nm}$. Therefore, this short wavelength is generally inefficient for reflectivity measurements. In general, it is better to change incident angle $\theta$ for NR measurements at high $q$ ranges.

\section{REFLECTIVITY MEASUREMENTS WITH STANDARD SAMPLES}

To quantitatively validate the SOFIA reflectometer, we carried out NR measurements on standard samples to ensure that the obtained results are consistent with known structural parameters such as SLD, thickness and characteristic $q$ values. The measurements in this section were performed at a proton power of $220 \mathrm{~kW}$.

Figure 4 shows the reflectivity profile of deuterated polystyrene (d-PS) spun-cast thin film on a silicon (Si) substrate $\left(30 \times 30 \mathrm{~mm}^{2}\right)$ at an angular resolution $\Delta \theta / \theta$ of $3.0 \%$. The solid line in the figure represents a best-fit curve based on model calculations assuming a three-layer model of $\mathrm{d}-\mathrm{PS} / \mathrm{SiO}_{2} / \mathrm{Si}$ (Figure $4 \mathrm{~b}$ ). The MOTOFIT program $^{12}$ combined with the Abeles matrix method ${ }^{13}$ was used to fit reflectivity profiles to the model SLD layers, wherein the thickness 
of each layer, SLD and Gaussian roughness were optimized to minimize the $\chi^{2}$ between the measured and calculated reflectivity curves. An identical result was obtained by the Parratt algorithm. ${ }^{14}$

The NR of d-PS film showed a total reflection at low $q$ ranges and several fringes between $q=0.2-1.2 \mathrm{~nm}^{-1}$. The observable reflectivity decreased by seven orders of magnitude over $q$ range of $0<q<4 \mathrm{~nm}^{-1}$. The obtained critical scattering vector $\left(q_{\mathrm{c}}\right)$ of the d-PS film was $0.18 \mathrm{~nm}^{-1}$, which was consistent with theoretical $q_{\mathrm{c}}$ values calculated by SLD of d-PS $\left(6.46 \times 10^{-4} \mathrm{~nm}^{-2}\right), \mathrm{SiO}_{2}(3.47 \times$ $\left.10^{-4} \mathrm{~nm}^{-2}\right)$ and $\mathrm{Si}\left(2.07 \times 10^{-4} \mathrm{~nm}^{-2}\right) .{ }^{15,16}$ The NR curve for d-PS thin film exhibits regular oscillation, so-called Kiessig fringes, with a constant period corresponding to film thickness. The fitting curve (solid line) in Figure 4a reproduced experimental NR plots very well up to $q \sim 2 \mathrm{~nm}^{-1}$ using a box model of d-PS (97.8 nm), $\mathrm{SiO}_{2}(1.25 \mathrm{~nm})$ and $\mathrm{Si}$ substrate. The thickness of d-PS estimated by NR was close to the thickness independently determined by ellipsometry $(98.2 \mathrm{~nm})$.
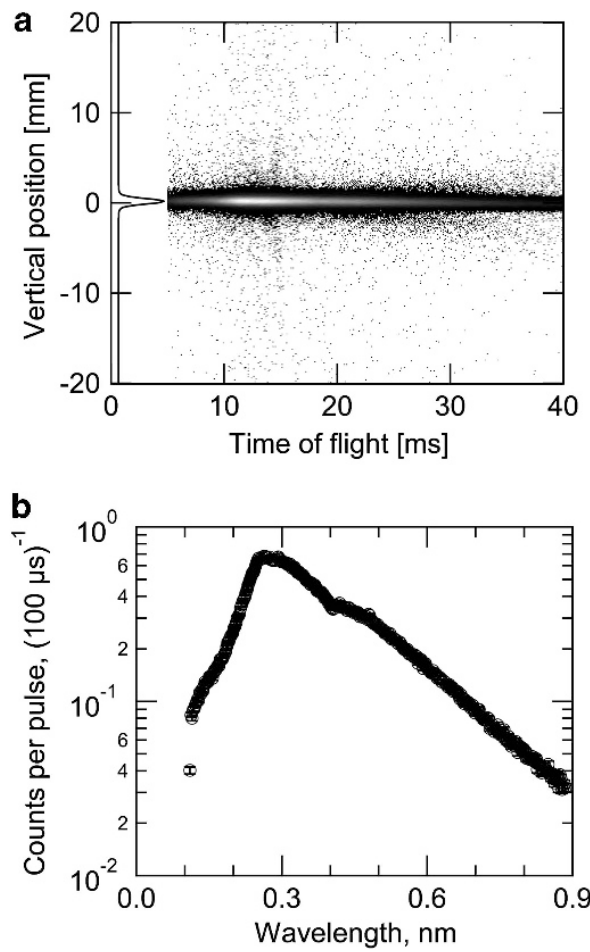

Figure 3 Intensity map of direct neutron beam (a) spatial distribution of intensity with time-of-flight (b) overall intensity versus wavelength.
In addition, the slope of the NR curve was gently changed at $q=1.0 \mathrm{~nm}^{-1}$, which was attributed to a very thin layer of $\mathrm{SiO}_{2}$ at the interface of d-PS and $\mathrm{Si}$. If the thin $\mathrm{SiO}_{2}$ layer was ignored in the fitting simulation, the theoretical reflectivity curve did not fit with the experimental NR curve at high $q$-region around $1-4 \mathrm{~nm}^{-1}$. The bestfitting curve was obtained by using the three box model of d-PS, $\mathrm{SiO}_{2}$ and $\mathrm{Si}$. The fitting simulations based on the absence and presence of the $\mathrm{SiO}_{2}$ thin layer are described in the Supplementary Information with detailed illustrations. This result indicates notable improvement in analysis of such a very thin layer by NR, owing to the supreme performance of the SOFIA.

Although the best-fit curve in Figure 4a reproduced experimental data over the entire range of $q$, the fringes at $q$ values larger than $1 \mathrm{~nm}^{-1}$ were unclear. Therefore, we performed another NR measurement of stacked bilayers of lipid up to $q=6 \mathrm{~nm}^{-1}$ and compared the Bragg peaks of the NR profile with those obtained from X-ray reflectivity (XR) using Rigaku R-AXIS (Rigaku, Tokyo, Japan) with a $\mathrm{Cu}-K_{\alpha} \mathrm{X}$-ray source. The lipid bilayers used here were cadmiumdeuterated stearate 7.5 bilayers, which were prepared on a Si substrate $\left(20 \times 30 \mathrm{~mm}^{2}\right)$ by the Langmuir-Blodgett method. The polar carboxyl acid cadmium salt were bound to the Si substrate, and the outer most surface of the film at the air interface was covered with the long alkyl chain of the stearyl group, as illustrated in Figure 5. If the LangmuirBlodgett film has a repetition of bilayers with spacing $d$, the Bragg peaks should be observed at neutron momentum transfer $q_{n}=2 \pi n / d$ $(n=1,2,3,4 \ldots)$ in specular neutron reflection.

Figures $5 \mathrm{a}$ and $\mathrm{b}$ show $\mathrm{NR}$ and XR curves of the cadmiumdeuterated stearate Langmuir-Blodgett film as a function of $q$, respectively. In the NR profile, four distinctive Bragg peaks were observed at $q=1.20,2.46,3.68$ and $4.91 \mathrm{~nm}^{-1}$, although the peak at $q=1.20 \mathrm{~nm}^{-1}$ was not as clear as the corresponding peak in the XR profile, probably because the contrast between SLD of cadmium ions and alkyl groups was lower than the corresponding contrast in electron density. The $q$ value at each peak position is listed in Table 1 together with corresponding $q$ values obtained from X-ray reflection. These Bragg peak positions obtained from NR are in good agreement with those of XR, within $\pm 5 \%$, which is comparable to the angular resolution of $3.0 \%$. The bilayers demonstrate that the diffraction peaks were fairly observable up to $q \sim 5 \mathrm{~nm}^{-1}$ on the SOFIA reflectometer.

Spacing $d$ of bilayers estimated from the Bragg peaks was $5.1 \mathrm{~nm}$, which was smaller than the bilayer sequence of non-tilted stearic acid $(5.5 \mathrm{~nm}),{ }^{17,18}$ indicating that the d-SA alkyl groups might be slightly tilted and oriented almost perpendicular to the film's surface. From the Kiessig fringe periodicity, the total film thickness obtained was
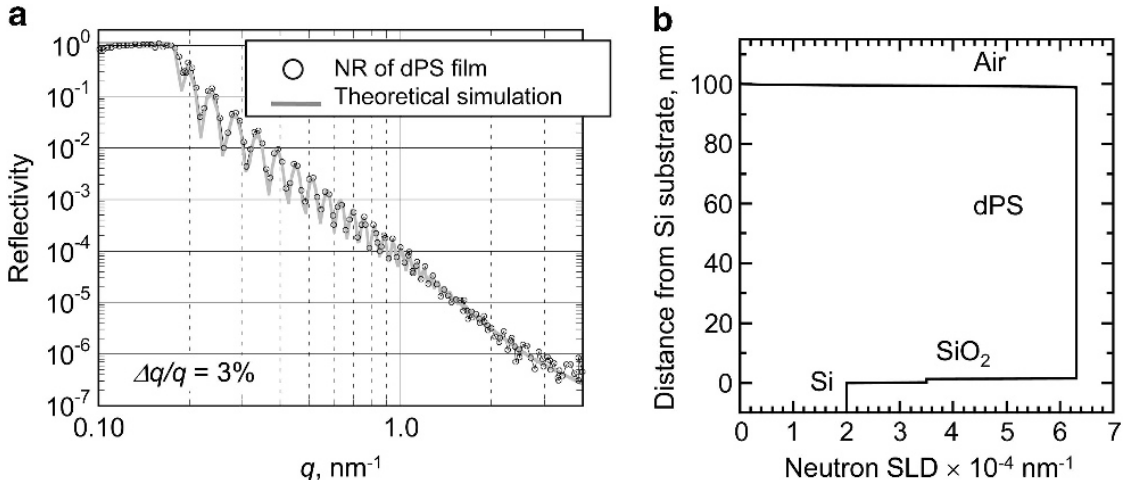

Figure 4 (a) NR profile of deuterated polystyrene (d-PS) thin film with the fitting curve and (b) the corresponding SLD profile. 


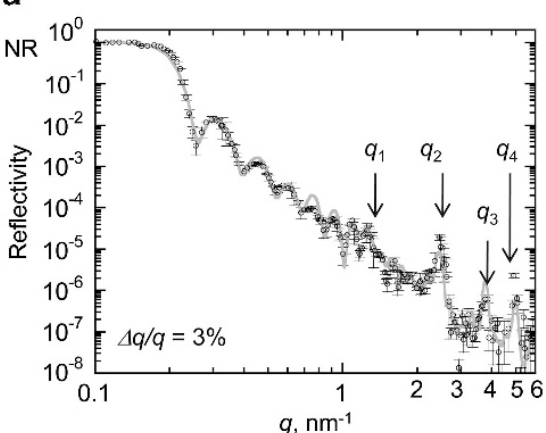

b

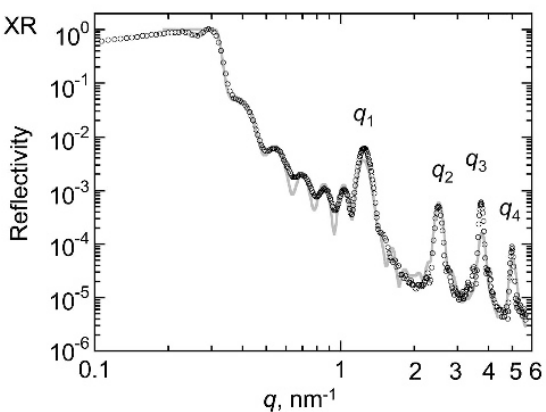

c

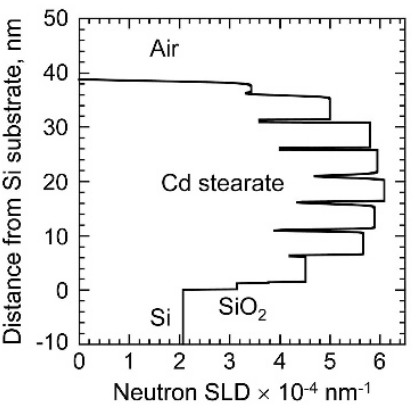

d

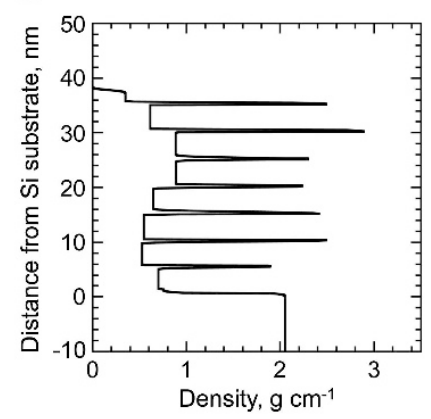

$\mathrm{l}=$ Cd stearate

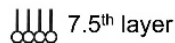

Figure 5 (a) NR, (b) XR, the corresponding (c) Neutron SLD, and (d) electron density profiles of 7.5 bilayers of cadmium stearate prepared on Si substrate by Langmuir-Blodgett method. NR profiles was obtained at $q$ resolution $(\Delta q / q)$ of $3 \%$. Peak positions are listed in Table 1.

Table 1 List of $q$ values obtained from NR and XR with Cd d-SA bilayers $^{\mathrm{a}}$

\begin{tabular}{lcccc}
\hline Layer, $\mathrm{n}$ & $\mathrm{q}_{n}(N R), n m^{-1}$ & $\mathrm{q}_{n}(X R), n m^{-1}$ & $\mathrm{~d}_{n}(N R), n m^{-1}$ & $\mathrm{~d}_{n}(X R), n m^{-1}$ \\
\hline 1 & 1.20 & 1.23 & 5.23 & 5.11 \\
2 & 2.46 & 2.47 & 5.11 & 5.09 \\
3 & 3.68 & 3.73 & 5.12 & 5.05 \\
4 & 4.91 & 4.99 & 5.12 & 5.03
\end{tabular}

Abbreviations: Cd d-SA, cadmium-deuterated stearate; NR, neutron reflectivity; XR, X-ray reflectivity.

asee Figure $4 ; d_{n}=2 \pi n / q$

$39 \mathrm{~nm}$. Using these parameters and the MOTOFIT program, we estimated the neutron SLD and electron density profiles of 7.5 bilayers from the NR and XR curves (Figures $5 \mathrm{c}$ and $\mathrm{d}$ ). The obtained SLD value of d-SA varied from 5.0 to $5.9 \times 10^{-4} \mathrm{~nm}^{-2}$ inside the film, except for the outermost 0.5 bilayer $\left(4.1 \times 10^{-4} \mathrm{~nm}^{-2}\right)$, although the theoretical SLD value of d-SA is $5.8 \times 10^{-4} \mathrm{~nm}^{-2}$ as calculated using a mass density of $0.84 \mathrm{~g} \mathrm{~cm}^{-3}$. The SLD of the first bilayer in contact with the Si substrate was relatively low in comparison with that of bilayers 2-7. The electron density of the cadmium ion layer near the Si substrate was also lower than that of the upper layers, probably due to the difference in molecular aggregation structure. Fujimori et al. ${ }^{19}$ reported that the cadmium fatty acid in the first bilayer on a solid substrate forms a hexagonally packed structure, whereas those in multilayers above brush are packed in a monoclinic state.

As a preliminary experiment, NR measurement at liquid/solid interfaces was demonstrated using deuterium oxide $\left(\mathrm{D}_{2} \mathrm{O}\right)$ and Si substrate. An aluminum trough was filled with $\mathrm{D}_{2} \mathrm{O}$ and covered with a Si disk ( $3^{\prime \prime}$ diameter, $10 \mathrm{~mm}$ thickness). A rubber O-ring was sandwiched between the trough and Si disk to prevent water leaks.
The incident neutrons were irradiated through the Si disk and reflected at the $\mathrm{D}_{2} \mathrm{O} / \mathrm{Si}$ substrate interface (Figure 6a).

As neutrons obey Snell's refractive law at the $\mathrm{Si} / \mathrm{D}_{2} \mathrm{O}$ interface with refractive indices $n_{0}(\mathrm{Si})$ and $n_{1}\left(\mathrm{D}_{2} \mathrm{O}\right)$, the relationship between the incident angle $\left(\theta_{0}\right)$ and refracted angle $\left(\theta_{1}\right)$ is described as follows:

$$
n_{0} \cos \theta_{0}=n_{1} \cos \theta_{1} .
$$

If total reflection occurs when incident angle $\theta_{0}$ is $\theta_{\mathfrak{c}}$, the angle of refraction $\theta_{1}$ is 0 .

$$
\cos \theta_{\mathrm{c}}=n_{1} / n_{0} .
$$

In general, the refractive index for nonmagnetic substances for neutrons, $n$, is given by

$$
n=1-\delta+i \beta
$$

where $\delta$ and $\beta$ are the real (dispersion) and imaginary (absorption) anomalous dispersion factors, respectively. ${ }^{1}$ However, $\beta$ can be regarded as 0 in most cases for neutrons. Real dispersion $\delta$ is related to the neutron $\mathrm{SLD}, \rho \Sigma b$, and is given by

$$
\delta=\left(\lambda^{2} / 2 \pi\right) \rho \Sigma b,
$$

where $\rho$ and $b$ are the atomic density and scattering length of the materials, respectively. Therefore, $n_{1}$ and $n_{0}$ can be expressed using SLD of $\mathrm{Si}$ and $\mathrm{D}_{2} \mathrm{O}$ :

$$
\begin{aligned}
& n_{0}=1-\left(\lambda^{2} / 2 \pi\right) \operatorname{SLD}_{\mathrm{Si}}, \\
& n_{1}=1-\left(\lambda^{2} / 2 \pi\right) \operatorname{SLD}_{\mathrm{D}_{2} \mathrm{O}},
\end{aligned}
$$

where $\operatorname{SLD}_{\mathrm{D}_{2} \mathrm{O}}=6.38 \times 10^{-4} \mathrm{~nm}^{-2}$ and $\mathrm{SLD}_{\mathrm{Si}}=2.07 \times 10^{-4} \mathrm{~nm}^{-2}$. Then, the critical value of momentum transfer, $q_{\mathrm{c}}$, along the $z$-direction for total reflection can be expressed as equation 8 , using 

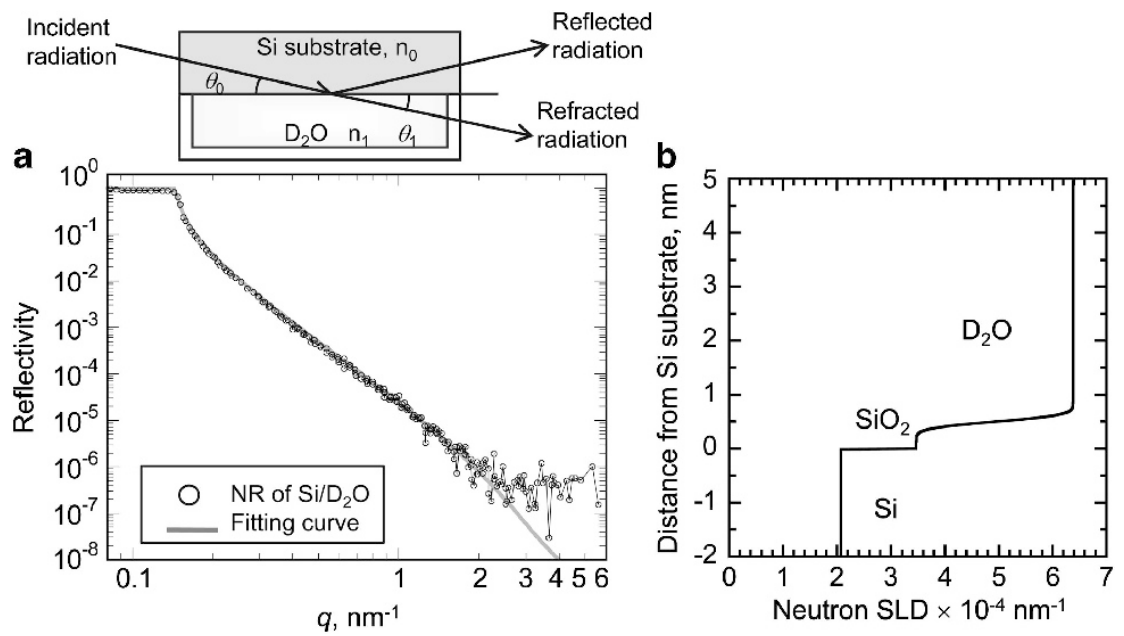

Figure 6 (a) NR profiles of $\mathrm{Si} / \mathrm{D}_{2} \mathrm{O}$ interface with the fitting curve and (b) the fitting model.

equations 3, 6 and 7:

$$
\begin{aligned}
q_{\mathrm{c}}= & (4 \pi / \lambda) \sin \theta_{\mathrm{c}}=(4 \pi / \lambda)\left(1-\left(n_{1} / n_{0}\right)^{2}\right)^{1 / 2} \\
= & (4 \pi / \lambda)\left\{\left(\lambda^{2} / \pi\right)\left(\mathrm{SLD}_{\mathrm{D}_{2} \mathrm{O}}-\mathrm{SLD}_{\mathrm{Si}_{\mathrm{i}}}\right)\right. \\
& \left.+\left(\lambda^{2} / 2 \pi\right)^{2}\left(\mathrm{SLD}_{\mathrm{Si}}^{2}-\mathrm{SLD}_{\mathrm{D}_{2} \mathrm{O}}^{2}\right)\right\}^{1 / 2} / n_{0} .
\end{aligned}
$$

Considering that $\mathrm{SLD}_{\mathrm{Si}^{2}}{ }^{2}$ and $\mathrm{SLD}_{\mathrm{D}_{2} \mathrm{O}}^{2}$ are sufficiently smaller than 1 and $n_{0} \sim 1$, respectively, $q_{\mathrm{c}}$ can be theoretically determined by

$$
q_{\mathrm{c}}=4\left\{\pi\left(\operatorname{SLD}_{\mathrm{D}_{2} \mathrm{O}}-\mathrm{SLD}_{\mathrm{Si}}\right)\right\}^{1 / 2}=0.147 \mathrm{~nm}^{-1} .
$$

As shown in Figure $6 \mathrm{a}, q_{\mathrm{c}}$, due to total reflection at the $\mathrm{D}_{2} \mathrm{O} / \mathrm{Si}$ interface, was observed at $0.146 \mathrm{~nm}^{-1}$, which was in excellent agreement with the predicted value.

The obtained reflectivity was well described by the corresponding fit (solid line in the figure) based on a $\mathrm{Si} / \mathrm{SiO}_{\mathrm{x}} / \mathrm{D}_{2} \mathrm{O}$ model (Figure $6 \mathrm{~b}$ ) up to $R \sim 10^{-7}$ and $q \sim 3 \mathrm{~nm}^{-1}$. Reflectivity with a magnitude lower than $10^{-7}$ was hardly obtained because of background noise, which might arise primarily from incoherent scattering from the bulk solution. Nonetheless, the results prove that the SOFIA reflectometer is capable of providing fair NR data over a wide $q$ range up to approximately $3 \mathrm{~nm}^{-1}$ even in a liquid/solid interface where the resulting maximum measurable $q$ range is sufficiently comparable to that obtained by the best NR instrument in the world. ${ }^{20-23}$

Typical accumulation times for NR measurements of air/d-PS thin film and $\mathrm{D}_{2} \mathrm{O} / \mathrm{Si}$ interfaces at a proton power of $220 \mathrm{~kW}$ are summarized in Table 2. Statistically sufficient counts for TOF neutrons for $q<1.0 \mathrm{~nm}^{-1}$ can be obtained within a few minutes from the air/d-PS interface on a Si wafer (76.2 mm diameter). The current accumulation time for the measurement is comparable to the best NR instrument overseas; however, NR measurement of a much smaller sample size and shorter timescale will be possible if $1 \mathrm{MW}$ operation of the proton beam starts in the near future. For the $\mathrm{Si} / \mathrm{D}_{2} \mathrm{O}$ interface, the measurements were finished within $2 \mathrm{~h}$ for the $q<2 \mathrm{~nm}^{-1}$ range. The short accumulation time obtainable by the combination of the SOFIA reflectometer and strong neutron source at J-PARC makes it far more feasible to perform the in-situ measurements described later in the manuscript.
Table 2 Typical accumulation time for NR measurements by SOFIA

\begin{tabular}{lccc}
\hline Sample $^{\mathrm{b}}$ & Size $^{\mathrm{c}}$ & q Range $\left(\mathrm{nm}^{-1}\right)^{\mathrm{d}}$ & Time \\
\hline Air/d-PS thin film on Si & $30 \times 30 \mathrm{~mm}^{2}$ & $<1$ & $10 \mathrm{~min}$ \\
Air/d-PS thin film on Si & $30 \times 30 \mathrm{~mm}^{2}$ & $<4$ & $2-3 \mathrm{~h}$ \\
Air/d-PS thin film on Si & $\phi 76.2 \mathrm{~mm}$ & $<1$ & $3 \mathrm{~min}$ \\
Air/d-PS thin film on Si & $\phi 76.2 \mathrm{~mm}$ & $<4$ & $1 \mathrm{~h}$ \\
$\mathrm{D}_{2} \mathrm{O} / \mathrm{Si}$ & $\phi 76.2 \mathrm{~mm}$ & $<2$ & $2 \mathrm{~h}$
\end{tabular}

Abbreviations: d-PS, deuterated polystyrene; NR, neutron reflectivity; SLD, scattering length density; SOFIA, SOFt Interface Analyzer, TOF, time-of-flight.

approximate time required to obtain statistically sufficient counts for TOF neutrons at proton power $220 \mathrm{~kW}$.

bSLD of d-PS, Si and $D_{2} \mathrm{O}$ are $6.46 \times 10^{-4}, 2.07 \times 10^{-4}$ and $6.43 \times 10^{-4} \mathrm{~nm}^{-2}$, respectively.

Size of silicon substrates.

dBeam divergence $\Delta \theta / \theta=5 \%$.

\section{NR OF A POLYMER BRUSH IN A SOLVENT}

Polymer brushes are surface-tethered polymers on a solid surface with sufficiently high grafting density and are relevant in colloid stabilization, wettability, antifouling, adhesion and tribology. A swollen or collapsed brush structure is responsible for surface behavior and for all of these applications. Therefore, it is important to characterize the brush height and segment density profile at the solid/liquid interface. The neutron reflection technique is quite useful for in-situ determination of static structures at the solid/liquid interface in nanometerlength scales. ${ }^{24-27}$ In our previous study, NR measurement of a swollen polymer brush in $\mathrm{D}_{2} \mathrm{O}$ was conducted using the ARISA-II reflectometer; however, the resulting specular reflection reached only $10^{-4}-10^{-5}$ in reflectivity because of high background noise and a $q$ range lower than $0.8 \mathrm{~nm}^{-1}$ due to the gradient density profile of the swollen brush structure. ${ }^{28}$ It was difficult to determine the swollen brush thickness and precise chain structure, because this kind of molecular information may be obtained at larger $q$ ranges than those accessible by ARISA-II.

As described in the previous section, the SOFIA reflectometer, in contrast, is capable of obtaining reflectivity up to $q=2.0 \mathrm{~nm}^{-1}$. Therefore, we again demonstrate NR measurement at the interface between $\mathrm{D}_{2} \mathrm{O}$ and the water-soluble polymer brush surface using the new reflectometer. A water-soluble poly[ $\{2$-(methacryloyloxy)ethyl $\}$ trimethylammonium chloride] (PMTAC) brush with $M_{\mathrm{n}}=110000$ 


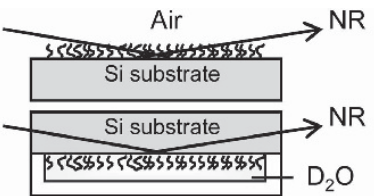

a

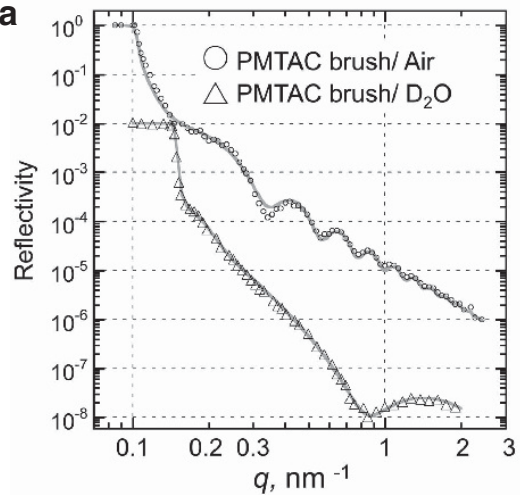

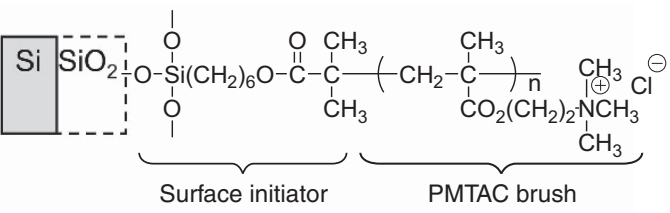

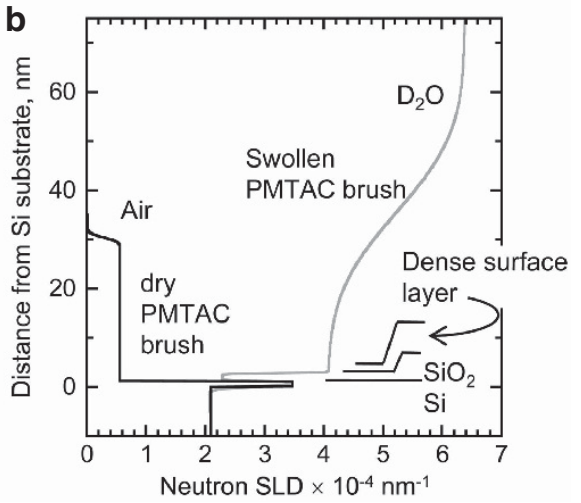

Figure 7 (a) NR curves of PMTAC brush interfaces in air (circle plot) and $\mathrm{D}_{2} \mathrm{O}$ (triangle plot), and (b) the corresponding neutron SLD profiles along with distance from the silicon surface.

and relatively narrow molecular dispersity $\left(M_{\mathrm{w}} / M_{\mathrm{n}}=1.19\right)$ was prepared by surface-initiated atom transfer radical polymerization from a Si disk with a $3^{\prime \prime}$ diameter and $10 \mathrm{~mm}$ thickness. ${ }^{29}$ Although the PMTAC is a hydrogenated polymer, structural analysis in polymer/deuterated solvent interfaces is possible due to the great SLD contrast enhancement between them. ${ }^{24}$

Figures $7 \mathrm{a}$ and $\mathrm{b}$ show NR curves of the PMTAC brush interfaces in air and $\mathrm{D}_{2} \mathrm{O}$, and the corresponding neutron SLD profiles along with the distance from the Si disk surface, respectively. Although the SLD of PMTAC is relatively low at $0.75 \times 10^{-4} \mathrm{~nm}^{-2}$ due to nondeuterated polymer, the thickness of brush film under air at 50\% relative humidity and $298 \mathrm{~K}$ was determined to be $29.0 \mathrm{~nm}$ from the periodic fringes, using a five-layer model (air/PMTAC brush/surface initiator layer $/ \mathrm{SiO}_{2} / \mathrm{Si}$.

On the other hand, NR of a PMTAC brush in $\mathrm{D}_{2} \mathrm{O}$ showed indistinguishable fringes; however, the specular reflection was measured down to $10^{-6}$ in reflectivity and up to $2.0 \mathrm{~nm}^{-1}$ in $q$, using white neutrons with wavelengths of $0.20-0.88 \mathrm{~nm}$. The SLD profile of the PMTAC brush in $\mathrm{D}_{2} \mathrm{O}$ was a smooth upward curve from $4.1 \times$ $10^{-4} \mathrm{~nm}^{-2}$ at the Si substrate surface to $6.38 \times 10^{-4} \mathrm{~nm}^{-2}$ at the $\mathrm{D}_{2} \mathrm{O} /$ brush interface. The SLD curve of the brush approached $\mathrm{SLD}_{\mathrm{D}_{2} \mathrm{O}}$ at ca. $50 \mathrm{~nm}$ from the surface.

In general, SLD of the swollen brush at a position $(z)$ apart from the substrate surface is determined by a volume fraction $\phi(z)$ of polymer brush in a solvent. Using SLD of MTAC and $\mathrm{D}_{2} \mathrm{O}, \operatorname{SLD}(z)$ can be expressed as

$$
\operatorname{SLD}(z)=\operatorname{SLD}_{\mathrm{MTAC}} \phi(z)+\operatorname{SLD}_{\mathrm{D}_{2} \mathrm{O}}(1-\phi(z))
$$

Figure 8 shows the $\phi(z)$ profile calculated by equation 9 based on the SLD of swollen PMATC brush in Figure 7b. According to the selfconsistent field theory, the chains near the grafting substrate are relatively confined compared with that at the exterior of the brush, resulting in the parabolic brush profiles. The $\phi(z)$ of densely grafted brush in a good solvent is represented by a following parabolic

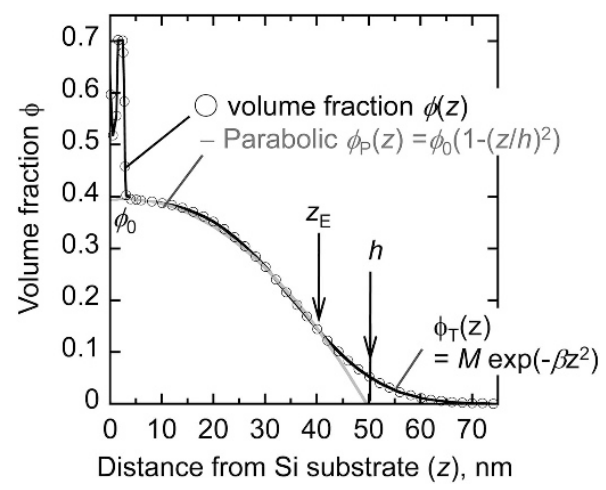

Figure 8 Volume fraction of PMTAC brush in $\mathrm{D}_{2} \mathrm{O}$ and the corresponding simulations using parabolic and Gaussian functions.

function $\phi_{\mathrm{P}}(z)^{30,31}$ and Gaussian tail $\phi_{\mathrm{T}}(z) ;^{32-35}$

$$
\begin{aligned}
& \phi(z)=\phi_{\mathrm{P}}(z)=\phi_{0}\left(1-(z / h)^{2}\right) \quad\left(0<z<z_{\mathrm{E}}\right) \\
& \phi(z)=\phi_{\mathrm{T}}(z)=M \exp \left(-\beta z^{2}\right) \quad\left(z_{\mathrm{E}}<z\right)
\end{aligned}
$$

where $\phi_{0}$ is the volume fraction at $z=0$, and $h$ is the cut-off thickness of the swollen brush layer when $\phi_{\mathrm{P}}(z)=0$, as shown in Figure 8 . The $\phi_{\mathrm{T}}(z)$ was expressed as Gaussian function using $M$ and $\beta$ as the parameters for the fitting of $\phi(z)$ at $z>z_{\mathrm{E}}$. As the $\phi_{\mathrm{P}}(z)$ and $\phi_{\mathrm{T}}(z)$ are connected at $z=z_{\mathrm{E}}$, the boundary condition are

$$
\mathrm{d} \phi_{\mathrm{P}}\left(z_{\mathrm{E}}\right) / \mathrm{d} z=d \phi_{\mathrm{T}}\left(z_{\mathrm{E}}\right) / \mathrm{d} z \text { and } \phi_{\mathrm{P}}\left(z_{\mathrm{E}}\right)=\phi_{\mathrm{T}}\left(z_{\mathrm{E}}\right)
$$

In this work, the volume fraction profile in $\mathrm{D}_{2} \mathrm{O}$ was in good agreement with a parabolic function with $h=50 \mathrm{~nm}$, indicating that the PMTAC chains in $\mathrm{D}_{2} \mathrm{O}$ were stretched up to ca. $50 \mathrm{~nm}$. Therefore, the polymer chains in $\mathrm{D}_{2} \mathrm{O}$ were relatively extended in the direction normal to the substrate.

However, the NR profile showed specular reflection at $q=0.8$ $2.0 \mathrm{~nm}^{-1}$, which indicated the presence of a dense layer of $2 \mathrm{~nm}$ thickness on the Si substrate surface, as shown in Figure $7 \mathrm{~b}$. The 
thickness of the dense surface layer is greater than that of the silane surface initiator $(0.9-1.0 \mathrm{~nm})$. PMTAC chains near the Si surface might be confined strongly to form the dense layer. ${ }^{36}$ We supposed that the PMTAC brush adopted a two-region structure consisting of some thin and dense surface regions and a brush region that stretches away from the substrate. The specular reflection at such a high $q$ range could not be previously observed at the $\mathrm{D}_{2} \mathrm{O}$ interface on the swollen brush by ARISA-II. Owing to the high flux and low background noise even at high $q$ ranges of the SOFIA reflectometer, the migrated thin layer with nanometer thickness at the solid/liquid interface was clearly observed.

\section{TIME-RESOLVED NR MEASUREMENT AT POLYMER INTERFACE}

In the previous section, we confirmed that precise structural information of the thin film could be obtained quantitatively by the SOFIA reflectometer using standard samples. This section describes the possibility of in-situ observation of the dynamic process of a polymer thin film, which is another function of the SOFIA reflectometer. We demonstrate here the interpenetration between the polymer brush and mobile (unbound) polymer chains, which is an interesting problem that involves the concepts of wet brush and dry brush (an unswollen or collapsed brush) ${ }^{37,38}$ by time-resolved NR measurement using the double-frame mode.

A bilayer thin film consisting of d-PS film and a hydrogenated PS (h-PS) brush was prepared on a Si wafer as follows: surface-initiated atom transfer radical polymerization of styrene on a Si substrate $\left(15 \times 40 \mathrm{~mm}^{2}\right)$ was carried out to produce a h-PS brush $\left(M_{\mathrm{n}}=92800\right.$ and $M_{\mathrm{n}} / M_{\mathrm{w}}=1.89$ ) with $96 \mathrm{~nm}$ thickness, which was determined by ellipsometry. The graft density was calculated to be 0.65 chains $\mathrm{nm}^{-2}$, by the equation $\left(\rho L N_{\mathrm{A}} / M_{\mathrm{n}}\right) \times 10^{4}$, where $\rho, L$ and $N_{\mathrm{A}}$ are the bulk density of PS, brush thickness and Avogadro's number, respectively. The d-PS $\left(M_{\mathrm{n}}=39500, M_{\mathrm{n}} / M_{\mathrm{w}}=1.07\right.$, thickness $\left.=95 \mathrm{~nm}\right)$ was spincoated on a separate Si wafer and carefully transferred to the water's surface by a floating method. ${ }^{39}$ The d-PS film floating on the water's surface was then lifted up using the h-PS brush Si substrate to form a d-PS/h-PS brush bilayer thin film, which was dried under vacuum at $298 \mathrm{~K}$ for $3 \mathrm{~h}$ to remove water. Thermal diffusion behavior of the d-PS chain into a PS brush thin layer was analyzed by in-situ, time-resolved NR measurements during annealing at $398 \mathrm{~K}$.

The NR curve of the bilayer film before annealing $(t=0 \mathrm{~min})$ showed clear fringes at $0.1<q<1.1 \mathrm{~nm}^{-1}$ (Figure 9a). The curve fitting for NR was conducted using MOTOFIT combined with a fivelayer model (air/d-PS layer/h-PS brush/SiOx/Si) as demonstrated in Figure 9b. The thicknesses of the d-PS and h-PS brushes were found to be 91 and $96 \mathrm{~nm}$, respectively. The interface width between the d-PS and h-PS brushes was smaller than $2 \mathrm{~nm}$.

The bilayer sample kept at room temperature was quickly transferred to the preheated thermal stage at $398 \mathrm{~K}$, covered with an aluminum plate, and then evacuated immediately. It took ca. 1 min to start the NR measurement after the heated sample stage was adjusted to the experimental position. NR data was continuously acquired every $1 \mathrm{~min}$. Figure 9a shows the NR curves of d-PS/h-PS brush film during annealing for $2,3,4,5,10$ and $15 \mathrm{~min}$.

In this study, NR measurement in a double-frame mode with incident beam angle $\theta$ of $1.15^{\circ}$ was carried out to obtain reflectivity at $0.14<q<2.0 \mathrm{~nm}^{-1}$ without any change in the sample stage angle, when the angular resolution $\Delta \theta / \theta$ was $5 \%$.

As shown in Figure 9a, the Kiessig fringes in the NR curves of the d-PS/h-PS brush film became smeared due to annealing because of the interfacial mixing of d-PS chains and the h-PS brush. The corresponding SLD profiles revealed that the increases in the total thickness of the film and the interface thickness at d-PS/h-PS by annealing at $398 \mathrm{~K}$ within a few minutes were due to thermal expansion. The interface thickness between the d-PS and h-PS brushes became $70 \mathrm{~nm}$ at $15 \mathrm{~min}$. Although the 1-min accumulation was not sufficient for the NR at a $q$ range greater than $0.5 \mathrm{~nm}^{-1}$, the structural change in the brush thin layer was clearly captured from the reflectivity at $q=0.1-0.5 \mathrm{~nm}^{-1}$ observed by time-sliced NR using the double-frame mode.

Therefore, time-resolved NR measurements on the SOFIA reflectometer combined with the double-frame mode are useful to investigate the real-time evolution of non-equilibrium physical processes such as dewetting, interfacial diffusion above a glass transition temperature ${ }^{39}$ and swelling behaviors of polymers contacting solvents or vapors. ${ }^{6-8}$ In particular, SOFIA is expected to follow the kinetics at the soft interfaces on a time scale of seconds by time-resolved NR when J-PARC starts full-power operation in the near future to increase the neutron flux.

\section{CONCLUSION}

We have installed a new neutron reflectometer SOFIA (at BL16 in J-PARC). The reflectometer is able to receive two downward neutron beams at $2.2^{\circ}$ and $5.7^{\circ}$, where incident neutrons with wavelengths of
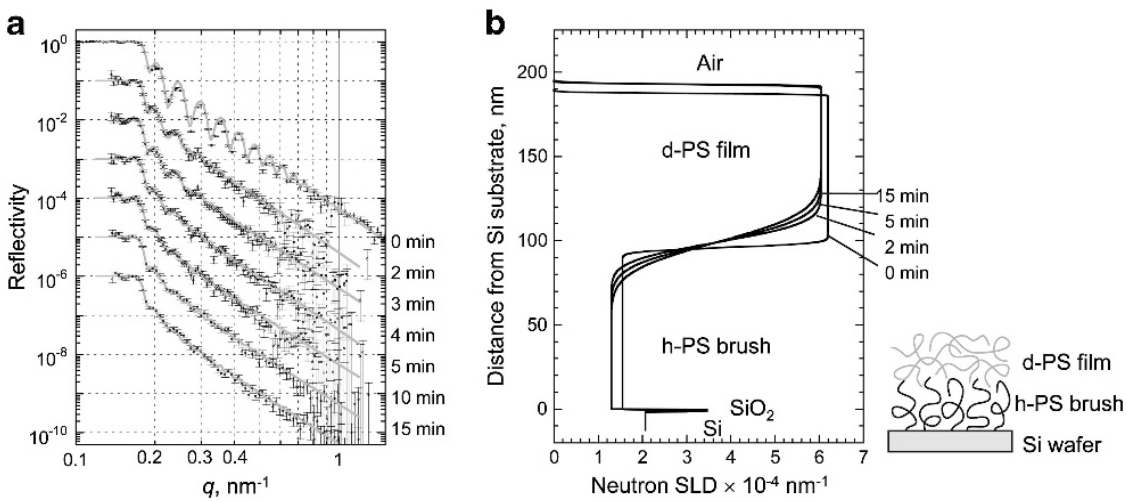

Figure $9 \mathrm{NR}$ profiles of d-PS $\left(M_{\mathrm{n}}=39500, M_{w} / M_{\mathrm{n}}=1.07\right) / \mathrm{h}$-PS brush $\left(M_{\mathrm{n}}=92800, M_{\mathrm{w}} / M_{\mathrm{n}}=1.89\right.$, graft density =0.65 chains nm $\left.{ }^{2}\right)$ bilayers before annealing $(t=0)$ and during annealing for (a) $t=2,3,4,5,10$ and $15 \mathrm{~min}$ under $398 \mathrm{~K}$, and (b) SLD profile of $t=0,2,5$ and $15 \mathrm{~min}$ after annealing. Reflectivity accumulation under $398 \mathrm{~K}$ started at $t=1 \mathrm{~min}$ after the sample was placed on vacuum hot stage. Reflectivity data were accumulated every 1 min by double-frame mode. 
$0.11-0.88 \mathrm{~nm}$ are provided by energy shaping with T0 and disk choppers in the single-frame mode. We confirmed the performance of SOFIA by several NR measurements. First, an NR curve of a d-PS thin film with $98 \mathrm{~nm}$ thickness on a Si substrate was successfully fitted with a theoretical curve. Second, NR of multilayer cadmium stearate showed that Bragg peaks originated from the periodic lamellar structure, whose $q$ values up to $4.9 \mathrm{~nm}^{-1}$ agreed very well with those obtained by X-ray reflectivity within an error $5 \%$. Finally, NR measurement at $\mathrm{D}_{2} \mathrm{O} / \mathrm{Si}$ substrate and $\mathrm{D}_{2} \mathrm{O}$ /polyelectrolyte brush interfaces showed specular reflection down to $10^{-6}-10^{-7}$ in reflectivity and up to $2.0 \mathrm{~nm}^{-1}$ in $q$, which provided precise analysis of the liquid/solid interface structure. Furthermore, time-resolved NR measurements at 1-min intervals using the double-frame mode successfully demonstrated the intermixing behavior of mobile d-PS and h-PS brushes during annealing at $398 \mathrm{~K}$. This technique must be useful for analysis of the structure change of soft interfaces on the order of minutes.

SOFIA will be further upgraded in the near future by installing focusing supermirrors to realize smaller and more intense beams without losing high resolution. This is because the grazing incident small angle neutron scattering measurements require a narrow beam along the grazing incident and perpendicular directions. Grazing incident small angle neutron scatterings have expanded the possibilities of NR towards studies for simultaneous characterization of the structural arrangement in the lateral as well as transverse directions at soft interfaces. We expect that the SOFIA reflectometer will provide an excellent opportunity to study surfaces, thin films and multilayers with its high flux, low background noise and very high resolution.

\section{ACKNOWLEDGEMENTS}

The construction of SOFIA was supported by Takahara Soft Interfaces Project of Japan Science and Technology Agency (JST), Exploratory Research for Advanced Technology (ERATO), Grant-in-Aid for Creative Scientific Research from the Ministry of Education, Culture, Sports, Science and Technology of Japan (16GS0417), and the S-type research project of KEK (2009S08).

1 Russell, T. P. X-ray and neutron reflectivity for the investigation of polymer. Mater. Sci. Reports 5, 171-271 (1990).

2 Kim, H., Rühm, A., Lurio, L. B., Basu, J. K., Lal, J., Lumma, D., Mochrie, S. G. J. \& Sinha, S. K. Surface dynamics of polymer films. Phys. Rev. Lett. 90, 068302 (2003).

3 Hoshino, T., Kikuchi, M., Murakami, D., Mitamura, K., Harada, Y., Ito, K., Tanaka, Y., Sasaki, S., Takata, M. \& Takahara, A. X-ray photon correlation spectroscopy of silica particles grafted with polymer brush in polystyrene matrix. J. Phys. Conf. Ser. 272, 012020 (2011).

4 Mezei, F. The principles of neutron spin echo. Lecture Notes in Physics 128, 1-27 (1980).

5 Dietrich, S. \& Haase, A. Scattering of X-rays and neutrons at interfaces. Phys. Reports 260, 1-138 (1995).

6 Krasteva, N., Krustev, R., Yasuda, A. \& Vossmeyer, T. Vapor sorption in self-assembled gold nanoparticle/dendrimer films studied by specular neutron reflectometry. Langmuir 19, 7754-7760 (2003)

7 Bucknall, D. G., Butler, S. A. \& Higgins, J. S. Real-time measurement of polymer diffusion coefficients using neutron reflection. Macromolecules 32, 5453-5456 (1999).

8 Ogawa, H., Kanaya, T., Nishida, K., Matsuba, G., Majewski, J. P. \& Watkins, E. Timeresolved specular and off-specular neutron reflectivity measurements on deuterated polystyrene and poly(vinyl methyl ether) blend thin films during dewetting process. J. Chem. Phys. 131, 104907 (2009)

9 Torikai, N., Furusaka, M., Matsuoka, H., Matsushita, Y., Shibayama, M., Takahara, A., Takeda, M., Tasaki, S. \& Yamaoka, H. Instrumental design and performance of a new pulsed-neutron reflectometer (ARISA) at KENS for studying free surfaces. Appl. Phys. A 74(Suppl 1), s264-s266 (2002).

10 Mitamura, K., Yamada, N. L., Sagehashi, H., Seto, H., Torikai, N., Sugita, T., Furusaka, M. \& Takahara, A. Advanced neutron reflectometer for investigation on dynamic/static structures of soft-interfaces in J-PARC. J. Phys. Conf. Ser. 272, 012017 (2011).

11 Yamada, N. L., Torikai, N., Mitamura, K., Sagehashi, H., Sato, S., Seto, H., Sugita, T. Goko, S., Furusaka, M., Oda, T., Hino, M., Fujiwara, T., Takahashi, H. \& Takahara, A.
Design and performance of horizontal-type neutron reflectometer SOFIA at J-PARC/ MLF. Eur. Phys. J. Plus 126, 108 (2011).

12 Nelson, A. Co-refinement of multiple-constant neutron reflectivity data using MOTOFIT. J. Appl. Crystal 39, 273-276 (2005).

13 Heavens, O. S. Optical Properties of Thin Films (Butterworth, London, 1955).

14 Parratt, L. G. Surface studies of solids by total reflection of X-rays. Phys. Rev 95 , 359-369 (1954)

15 Sears, V. F. Neutron scattering lengths and cross sections. Neutron News 3, 26-37 (1992).

16 Torikai, N. Neutron Reflectometry. in Neutrons in Soft Matter (eds Imae, T., Kanaya, T., Fususawa, M. \& Torikai, N.) 115-145 (Jon Wiley \& Sons, Inc, Hoboken, NJ, 2011).

17 Rothberg, L., Higashi, G. S., Allara, D. L. \& Garoff, S. Thermal disordering of LangmuirBlodgett films of cadmium stearate on sapphire. Chem. Phys. Lett. 113, 67-72 (1987)

18 Englisch, U., Peñacorada, F., Brehmer, L. \& Pietsch, U. X-ray and neutron reflection analysis of the structure and the molecular exchange process in simple and complex fatty acid salt langmuir-blodgett multilayers. Langmuir 15, 1833-1841 (1999)

19 Fujimori, A., Sugita, Y., Nakahara, H., Ito, E., Hara, M., Matsuie, N., Kanai, K., Ouchi, Y. \& Seki, K. Change of molecular packing and orientation from monolayer to multilayers of hydrogenated and fluorinated carboxylates studied by in-plane $X$-ray diffraction together with NEXAFS spectroscopy at C K-edge. Chem. Phys. Lett. 387, 345-351 (2004).

20 Penfold, J., Richardson, R. M., Zarbakhsh, A., Webster, J. R. P., Bucknall, D. G., Rennie, A. R., Jones, R. A. L., Cosgrove, T., Thomas, R. K., Higgins, J. S., Fletcher, P. D. I., Dickinson, E., Roser, S. J., McLure, I. A., Hillman, A. R., Richards, R. W. Staples, E. J., Burgess, A. N., Simistero, E. A. \& White, J. W. Recent advances in the study of chemical surfaces and interfaces by specular neutron reflection. J. Chem. Soc. Faraday Trans. 93, 3899-3917 (1997).

21 Campbell, R. A., Wacklin, H. P., Sutton, I., Cubitt, R. \& Fragneto, G. FIGARO: the new horizontal neutron reflectometer at the ILL. Eur. Phys. J. Plus 126, 107 (2011).

22 Cousin, F., Ott, F., Gibert, F. \& Menelle, A. EROS II: a boosted time-of-flight reflectometer for multi-purposes applications at the Laboratoire Leon Brillouin. Eur. Phys. J. Plus 126, 109 (2011).

23 Dubey, M., Jablin, M. S., Wang, P., Mocko, M. \& Majewski, J. SPEAR - ToF neutron reflectometer at the Los Alamos Neutron Science Center. Eur. Phys. J. Plus 126, 110 (2011).

24 Perahia, D., Wiesler, D. G. Satija, S. K., Fetters, L. J., Sinha, S. K. \& Milner, S. T. Neutron reflectivity of end-grafted polymers: concentration and solvent quality dependence in equilibrium conditions. Phys. Rev. Lett. 72, 100-103 (1994).

25 Anastassopoulos, D. L., Vradis, A. A., Toprakcioglu, C., Smith, G. S. \& Dai, L. Neutron reflectivity study of end-attached telechelic polymers in a good solvent. Macromolecules 31, 9369-9371 (1998).

26 Currie, E. P. K., Wagemaker, M., Cohen Stuart, M. A. \& van Well, A. A. Structure of grafted polymers, investigated with neutron reflectometry. Physica. B. 283, 17-21 (2000)

27 Marzolin, C., Auroy, P., Deruelle, M., Folkers, J. P., Leger, L. \& Menelle, A. Neutron reflectometry study of the segment-density profiles in end-grafted and irreversibly adsorbed layers of polymer in good solvents. Macromolecules 34, 8694-8700 (2001).

28 Kobayashi, M., Mitamura, K., Terada, M., Yamada, N. L. \& Takahara, A. Characterization of swollen states of polyelectrolyte brushes in salt solution by neutron reflectivity. J. Phys. Conf. Ser. 272, 012019 (2011).

29 Kobayashi, M., Terada, M., Terayama, Y., Kikuchi, M. \& Takahara, A. Direct synthesis of well-defined Poly[\{2-(methacryloyloxy)ethyl\}trimethyl ammoniumchloride] brush via surface-initiated ATRP in fluoroalcohol. Macromolecules 43, 8409-8415 (2010).

30 Milner, S. T., Witten, T. A. \& Cates, M. E. Theory of the grafted polymer brush. Macromolecules 21, 2610-2618 (1988).

31 Migliorini, G. On the corrections to strong-stretching theory for end-confined, charged polymers in a uniform electric field. Macromolecules 43, 9168-9180 (2010).

32 Sanjuan, S., Perrin, P., Pantoustier, N. \& Tran, Y. Synthesis and swelling behavior of ph-responsive polybase brushes. Langmuir 23, 5769-5778 (2007)

33 Karim, A., Satija, S. K., Douglas, J. F., Ankner, J. F. \& Fetters, L. J. Neutron reflectivity study of the density profile of a model end-grafted polymer brush: influence of solvent quality. Phys. Rev. Lett. 73, 3407-3410 (1994).

34 Field, J. B., Toprakcioglu, C., Ball, R. C., Stanley, H. B., Dai, L., Barford, W., Penfold, J., Smith, G. \& Hamilton, W. Determination of end-adsorbed polymer density profiles by neutron reflectivity. Macromolecules 25, 434-439 (1992).

35 Weir, M. P., Heriot, S. Y., Martin, S. J., Parnell, A. J., Holt, S. A., Webster, J. R. P. \& Jones, R. A. L. Voltage-induced swelling and deswelling of weak polybase brushes. angmuir 27, 11000-11007 (2011).

36 Dunlop, I. E., Thomas, R. K., Titmus, S., Osborne, V., Edmondson, S., Huck, W. T. S. \& Klein, J. Structure and collapse of a surface-grown strong polyelectrolyte. brush on sapphire. Langmuir 28, 3187-3193 (2012).

37 de Gennes, P. G. Conformations of polymers attached to an interface. Macromolecules 13, 1069-1075 (1980)

38 Ferreira, P. G., Ajdari, A. \& Leibler, L. Scaling law for entropic effects at interfaces between grafted layers and polymer melts. Macromolecules 31, 3994-4003 (1998).

39 Kawaguchi, D., Tanaka, K., Takahara, A. \& Kajiyama, T. Surface mobile layer of polystyrene film below bulk glass transition temperature. Macromolecules $\mathbf{3 4}$ 6164-6166 (2001).

Supplementary Information accompanies the paper on Polymer Journal website (http://www.nature.com/pj) 